\title{
Exploring factors influencing the retention rates of Indigenous students in post-secondary education
}

\author{
Sherry Ann Arvidson, Cheyanne Desnomie, Shauna Davies, Florence Luhanga \\ Faculty of Nursing, University of Regina, Canada
}

Received: June 3, 2020

Accepted: July 7, 2020

Online Published: July 24, 2020

DOI: $10.5430 /$ jnep.v10n11p24

URL: https://doi.org/10.5430/jnep.v10n11p24

\begin{abstract}
In comparison to Caucasian students, Indigenous students are outnumbered when it comes to enrollment in post-secondary education programs. Designated seats for Indigenous students often sit empty. With an aim to succeed academically, Indigenous students have had to develop a strong sense of resiliency and identity to overcome barriers to attend institutions of higher learning. Questions still remain as to why the seats are not being filled or what is preventing Indigenous students from enrolling in post-secondary education resonate among faculty and administrative leaders. Tinto's model of persistence confirmed the importance of integrating social involvement in academia. Students need support to achieve academic success and personal satisfaction. Motivational factors consisting of specific family member encouragement and exploring a better way of life was seen as the main reason to enroll in post-secondary education. Limitations of support at the peer and institutional levels were seen as challenging for Indigenous students and often times had an impact on academic completion. Questions as to why the seats are not being filled or what is preventing Indigenous students from enrolling in higher education programs led to the purpose of this study.
\end{abstract}

Key Words: Indigenous education, Success, Determinants of health, Community, Persistence, Cultural leadership

\section{INTRODUCTION}

Indigenous peoples are one of the fastest-growing demographic populations in Canada. It is estimated by 2050 that the Indigenous population in Saskatchewan will range from 35 to 50 percent of the total population. ${ }^{[1]}$ This rapid growth in population highlights the need for the development of programming to ensure that Indigenous students succeed in places of higher learning. The following study, which took place at a university in central Canada, found that the few Indigenous students who enrolled in post-secondary education have had to develop a strong sense of Identity, community belonging, and knowledge of resources as they are often confronted with challenges to further their education beyond grade school. Barriers in post-secondary education often included financial issues and lack of support and encourage- ment. ${ }^{[2-4]}$ While the majority of participants in this study did not report experiencing an overwhelming amount of racism in their programs, a number of them did report having to stand up for Indigenous issues and/or experienced culture shock when arriving at the institution. Issues resulting from institutional and systemic ideology were also mentioned. Financial struggles, coping with family member addictions, and childcare issues were contributing factors.

This research project led researchers to explore the reasons behind the factors influencing the retention rates of Indigenous students in three undergraduate programs at a postsecondary institution in central Canada. Students from the Faculties of Nursing, Social Work, and Education were invited to participate in the study. The study took place over

*Correspondence: Sherry Ann Arvidson; Email: sherry.arvidson@uregina.ca; Address: Faculty of Nursing, University of Regina, Canada. 
a two-year period. Data were collected until there was a lack of interest from participants and until the point of data saturation. Four themes evolved and represent the important elements for student success and academic achievement. The findings best represent the factors that influence a student's ability to perform well in academia and achieve a positive learning outcome.

\subsection{Purpose}

The purpose of the study was to identify factors influencing the retention rates of Indigenous students in a post-secondary Canadian institution. Social determinants of health and traumatic events have led to barriers among Indigenous peoples throughout Canadian history. ${ }^{[5]}$ Colonialism, the residential school system, and missing and murdered Indigenous women were a few events, which have impacted the lives of Indigenous peoples. ${ }^{[5]}$ Determining the factors affecting students in post-secondary programs is important to provide and promote student success and achievements. The study was driven by three questions seen as important elements in constructing new strategies and developing improvements for student success.

\subsection{Research questions}

The primary questions in the research project stem from concerns surrounding a perceived lack of Indigenous presence in various faculties at the University.

RQ1: What are the retention rates of Indigenous students in larger Faculties such as Social Work, Nursing and Education?

RQ2: What are the factors influencing the retention rates of Indigenous students?

RQ3: What support systems do Indigenous students utilize on campus?

\section{METHODOLOGY \& RESEARCH DESIGN}

Initially, the idea of a sequential mixed-methods study was of interest and deemed suitable for the study. Researchers planned for the collection of quantitative data from a demographic survey and questionnaire. After close examination of the data, the expectation was that an analysis of data from face-to-face interviews would complement the findings. Realizing that the quantitative portion did not receive sufficient attention, the research team decided to focus on the qualitative portion and collected data from face-to-face interviews until the point of data saturation was achieved. The quantitative portion became a limitation to the project due to an insignificant number of respondents. A phenomenological approach was used to explore the lived experiences of the participants and to allow for individual thoughts and expression Published by Sciedu Press of feelings. Despite the low number of respondents, utilizing a mixed-methods approach would have allowed for the opportunity to include marginalized voices in the research. ${ }^{[6,7]}$ Unlike the use of a mixed-methods design used by Botha in 2012, a reflection on the experiences of participants through a social lens could not be quantified. ${ }^{[6]}$

The conceptual framework of social influences and academic success fit well with the study and was used to analyze the data findings. Understanding the connection between student success and social influences were represented through the work on Tinto's model of academic persistence. ${ }^{[8]}$ A descriptive approach that focused on lived experiences and thoughts seemed to have a better reflection from a cultural view. Considering the values of the Indigenous people, including the ethical and relational aspects of knowledge is essential to draw out meaningful information used in research. This process is important as it allows for an enhanced understanding of the data. ${ }^{[9]}$ Research involving Indigenous peoples cannot only reveal knowledge but also decolonize, rebalance power, and provide healing. ${ }^{[10]}$ The inclusion of Indigenous researchers in the research process was a benefit to the entire team. Historically, this inclusion of Indigenous peoples to partake in decision-making processes was denied. ${ }^{[9]}$

\subsection{Population criteria and sampling}

Students were undergraduates enrolled as either part-time or full time from the Faculties of Social Work, Nursing, or Education. Considerably larger faculties were chosen for the study to capture experiences from the students. Students self-declared as Indigenous and were either living on or offreserve. The study was open to students in years one to four of a degree program. Students completed a demographic survey and a questionnaire, both of which were available online. A face-to-face semi-structured interview was available for students to share their experiences attending a post-secondary program. Students made connections with the importance of academic success and cultural recognition. Highlights of the study include the findings, conclusions, and recommendations to support student success with an emphasis on promoting Indigenous culture.

\subsection{Conceptual frameworks: Persistence and resilience}

Tinto ${ }^{[11]}$ identified that academic engagement alone is not enough to help some students persist. Social integration and involvement provide a counterbalance. Participation in social functions, extracurricular events seemed to have a connection with higher student success. Achievement in the classroom can be obtained through continual persistence. Early intervention is crucial for individuals to experience success. ${ }^{[3]}$ Feelings of accomplishment can further take place from the 
improvement of classroom strategies. Four strategies identified by Tinto include: Expectations, support, assessment and feedback, and involvement. ${ }^{[4]}$ Setting ground rules in the classroom may be one way to guide students to develop realistic goals and a plan for learning strategies that will help with the challenges faced in post secondary education. Providing students with clear and detailed information will assist students to transition into the learning environment and prepare for academic success.

\subsubsection{Relevance of persistence on research study}

Supporting students with learning is important in an environment that is known to have high standards. For minority populations, academic success can be challenging. ${ }^{[11]}$ Tinto argued that support is most effective when it directly aligns with daily needs of the learner. The need for support from a social aspect is equally important. To implement the need of social support, various events and cultural networks may be an option for students experiencing struggles with course work. Faculty can also play an active role by providing ongoing formative feedback throughout the term. Regular monitoring of student progress can keep students informed of their progress and provide faculty with an opportunity to alter their actions based on the needs of the student.

Providing students with feedback establishes an environment of self-reflection and encourages critical thinking. Midterm grades can be interpreted as a time to inform students of academic progress and may serve as a target to identify the need for additional instruction or additional support. Lastly, involvement in the classroom may have a significant influence on student success and persistence. Factors influencing student success may include the need to build a sense of community and recognition. Relevant to the study findings, course could implement an Indigenous community model that focuses on strategies for student success and social involvement. Doing so would also create a set of assets and resources for students to utilize and develop, such as a sense of identity, belonging and confidence among their chosen faculties. Strategies that foster critical thinking may include cooperative learning, problem-based learning, or project-based learning methods of instruction. These strategies encourage student interactions and relationship building. In addition, these learning strategies can be used in the development of critical skills.

In addition to Tinto's framework, Zimmerman ${ }^{[12]}$ has also published valuable resources that can be applied to Indigenous learners in higher education. Specifically, Zimmerman discusses the idea of the resilience theory and the need to focus on the positive, contextual, social, and individual variables that interfere with or abrupt developmental trajectories.
For instance, Zimmerman recommends focusing on assets and resources available to individuals. Positive factors such as self-efficacy, self-esteem and resilience are assets beneficial to individuals. In contrast, resources are factors outside of the individual such as parental support, adult mentors, peer support or youth programming. ${ }^{[12]}$ Focusing on developing these assets and resources within the academy and the faculties of social work, nursing and education could increase retention and success rates among Indigenous students.

\subsection{Evolving themes}

The four key themes were supported with additional subthemes, as evidenced by the exchange of participant experiences and knowledge. Themes include Identity, community, kinship and support. An illustration of the data is displayed in the form of a medicine wheel, which reflects the spiritual meaning of the Indigenous heritage (see Figure 1).

\subsubsection{Theme 1: Identity}

Themes emerged indicative of the importance of Identity, both culturally and from an occupational perspective. It was important for the participants to have a sense of Identity; however, many participants felt a real disconnect with their sense of identity or Indigenous heritage. In a discussion on racial Identity, it was noted that a strong sense of race and cultural identity might contribute positively to one's academic performance. ${ }^{[13]}$ Occupational Identity also emerged from the data as some participants described their preferred career as a calling.

\subsubsection{Theme 2: Community}

The community was seen as an important concept and shaped an individual's view on determining inclusion in processes and decision-making. A community can be made up of various factors such as cultural, academic, family and friends. Participants recognized the importance of giving back to their Indigenous communities in their future professions. Participants shared the importance of participating in activities, such as organized sports, events, or student groups, as they were able to find someone with the same passions to help with the transition to university life.

\subsubsection{Theme 3: Kinship}

Kinship often was identified from role models, family, and friends. Intergenerational effects have impacted each student in the study. Factors such as residential schools, addiction issues or family encouragement to pursue secondary education all affected the goals that the participants made in order to create a positive future for themselves and their own children. 


\subsubsection{Theme 4: Support}

Support was identified as essential to be successful in academia. Knowing where to go, who to speak with and how to access supports is critical to help ease students with the transition period to secondary education. Understanding the importance of support and social inclusion proved to be a determining factor in academic success and is best represented through the conceptual framework by Tinto. ${ }^{[11]}$ Participants felt it was important for the post-secondary institution to take into consideration student's needs related to family, home-life academic and extracurricular activities.

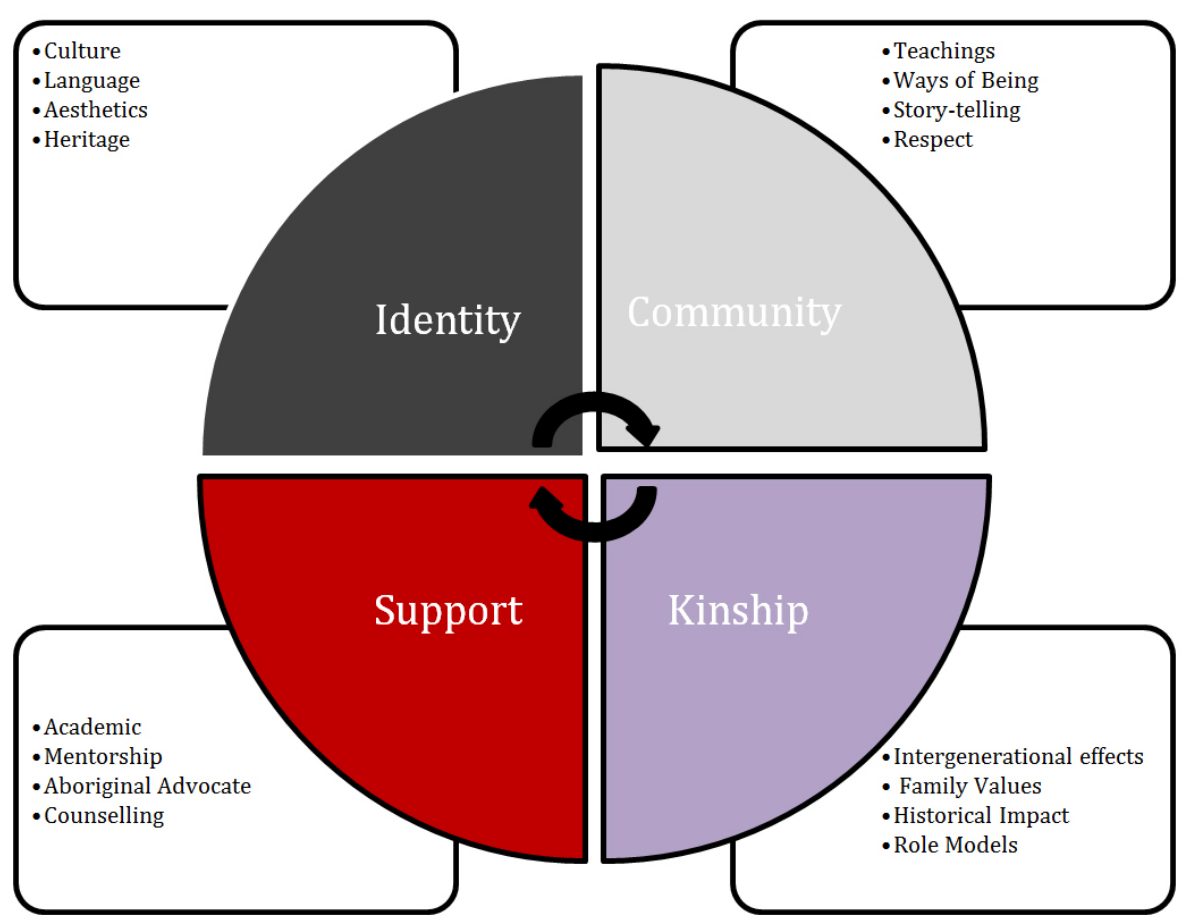

Figure 1. Identified themes for student success

\section{FINDings}

The findings are significant to the educational programs and University at large. Expressions of cultural awareness and improvements with Indigenous cultural events were the main areas identified by the participants as lacking, inadequate, and minimally visible. Students relied on Indigenous program advisors for academic support; however, university resources and events significant of Indigenous traditions were referred to as non-existent. Participants often turned to Elders for spiritual guidance. Smudging activities took place to help students with focusing on workload expectations. Tipi raising events were seen as an event that brought students together to celebrate Indigenous culture.

Interestingly, the majority of students interviewed were in their first year of post-secondary education. Students said they took part in cultural events on campus but lacked a feeling of confidence and understanding behind their own Indigenous culture. Often, the role model for the participants was their "Kokum" - (grandmother). Participants stated they did not have a strong connection with the Indigenous culture

Published by Sciedu Press because of their cultural background self-declared as Metis. Some expressed concerns was that living in a house with two different cultures led to a feeling of disconnect and a feeling of being torn between two very different cultures; however, their role model, usually "Kokum" influenced their desire to pursue post-secondary education. Kokum was seen as an important and highly respected individual in the Indigenous culture. Other recognized and important role models often included a relative who was of the female gender. The participants not only identified a female figure, but they also made a connection as to why the specific person was considered of such value. An aunt, sister, or mother was identified as people having "wisdom." Several participants stated they were participating in the study with their Kokum in mind. "This is something my Kokum would be proud of."

\subsection{Obstacles and barriers to academic success}

Indigenous students face unique barriers in comparison to non-Indigenous students. ${ }^{[3]}$ As faculty, it is important to recognize that Indigenous students may perform differently due to acts of colonization and the long-term effects of the resi- 
dential school system. Historical impacts of Canada's Residential School System identified students as being subjects of verbal, physical, and sexual abuse as victims. ${ }^{[4]}$ Although the residential schools closed in the 1990s, their impacts still resonate. Emotional and psychological damage was one of the most devastating legacies of residential schools. ${ }^{[5]} \mathrm{Im}-$ pacts on Indigenous cultures, traditions and languages have created a sense of distrust in formal education systems. In previous research, systemic and institutionalized racism has been identified as a contributing factor to the lack of retention and success of Indigenous students. ${ }^{[14]}$ Unlike the findings in this study, students did not explicitly state that racism was a barrier to their educational experience. When analyzing the data gathered through interviews, several statements revealed that institutional racism was present.

The direct and indirect effects had potential results on an individual's academic preparedness for higher education. ${ }^{[15]}$ In addition to educational impacts, the nature of assimilation may have led some Indigenous students to feel that they do not belong at post-secondary institutions. This particular finding was verbalized by a student who indicated a feeling of not being welcomed and as a result, led to the transfer to another program at a different university.

\subsection{Experience gained}

The research project was a learning opportunity for both researchers and participants. Additional training and workshops helped to understand the ethics underlying Indigenous research, the data collection process, the importance of an Elder, and the process for the dissemination of information through an Indigenous lens. Before undertaking the research project, the research team consulted with the late Noel Starblanket, a prominent knowledge keeper in the Indigenous community. For non-Indigenous researchers, an Indigenous focus allowed for the researchers to enter into the world alongside the Indigenous experience rather than framing the Indigenous worldview from a distance. ${ }^{[9]}$

Collaboration between members on the research team led to understanding the importance of cultural identity and sensitivity. The plan to have an Elder at the beginning of the study was to build a working relationship among members on the research team and to identify the need to support the researchers in finding ways to improve learning for Indigenous students in post-secondary education. Presenting the Elder with tobacco would signify trust and give thanks for the spiritual inclusiveness of the project. Developing relationships not only demonstrates an interest in one's Identity but also allows for an opportunity for meaningful learning to transpire.
Building working relationships is essential and strengthens the trust between people of various cultures. ${ }^{[9]}$ Similar to the participants in the study, developing trust was an essential part of feeling confident and comfortable with one's cultural traditions and practices. Once an adequate level of trust was established, members experienced openness and sensed a comfortable environment of meaningful interaction. The research team consisted of six members, with two of the six members who self-identified as Indigenous. Likewise, the participants and researchers both worked together to develop similar relationships that fostered openness and freedom during the exchange of information. ${ }^{[16]}$

Metis students reported that they did not feel comfortable with traditional practices. Many of the same students mentioned that they would like to attend cultural events but have been removed from family cultural traditions that attending the events would not be meaningful. Responses from First Nation students included feeling unwelcomed and excluded at the current University. As a result, these students either transferred to a different program to put their experience of exclusion behind or transferred to another post-secondary institution where they described as a better fit.

Developing trusting relationships is essential for researchers and especially significant for Indigenous people. The length of time that it takes to develop a trusting environment varies; however is not to be rushed or forced in any way. ${ }^{[2]}$ Promoting an environment with a high level of trust resulted in elaborative and meaningful data. Confirmation of the various levels of trusting relationships was experienced during the interview portion of the research study. Participants that were interviewed spent time with an introduction of the research study and a pre-interactive phase of getting to know each other. In some instances, this relationship lasted up to two hours. When the pre-interactive phase was short, the amount of information collected was minimal and had less meaning. Prior to signing the consent form for the interview, the trusting relationship transpired. At this phase, participants felt comfortable, were relaxed, and open to respond to the questions asked. Participants' level of trust had a great significance on the connection of cultural influences and the exchange of information.

\subsection{Limitations}

The study was limited to the 2017 and 2018 academic year from a single university in central Canada and, therefore, cannot be generalized beyond that scope. Only students from three undergraduate level programs were chosen for the study. Students who recently dropped out of the program were not considered as participants for the study. By adhering to the interview script, the wording of the questions limited the 
participants to expand on the questions.

Researchers were engulfed in a mixed-method sequential design at the beginning of the project; however, limitations in the number of respondents did not warrant significance from a quantitative perspective and as such, the study became central to having a qualitative focus. Additional training on Indigenous culture and historical events may have created a better understanding of the challenges that people faced. Having a mix of Indigenous and non-researchers on the team and an Elder to give blessings may have developed a closer working relationship for the researchers. Trust seemed to be an element present between Indigenous participants and nonIndigenous researchers. Disclosure of information may have prevented students from disclosing valuable and meaningful information for the study. The location of the interviews may have prevented students from feeling welcomed or taken away from their comfort zone. As with events involving knowledge exchange, students were gifted with a card of monetary value according to the researcher's budget. Additional strategies to make the participants feel more welcomed could have been made available such as having one of the Indigenous researchers available to conduct the interviews.

\subsection{Recommendations}

In higher education, leaders and administrators should work diligently to provide opportunities for students to get involved with campus activities and events. Involvement in community events and giving back through service may be an advantage for marginalized people. ${ }^{[9]}$ Supporting learning and engagement is important for Indigenous students to feel welcomed and appreciated. ${ }^{[17]}$ Creating awareness and offering information on available events demonstrates willingness and efforts to make a difference in the lives of people who deserve fairness and appreciation. Feedback from participants included developing a support centre for students enrolled in specific programs such as nursing support. This program could be seen as an area where Indigenous students in their senior year develop and lead activities by offering information to first-year students accepted into the nursing program. Starting early and creating a welcoming atmosphere through orientation events could build a trusting environment while focusing on academic support.

Recommendations for future research may include the following: Broadening the scope of the study to include other institutions in other areas of Canada, enlarge the study to include graduate students, promoting the integration of traditional events, and having an Elder available for classes was seen as crucial for spiritual and emotional support. Reaching out to students demonstrates support from various perspectives and looks at an individual holistically.

\section{Conclusions}

Students who are involved and engaged in sports and extracurricular activities at their institution are more likely to experience success in the classroom and complete their academic studies. Based on the importance of student support, institutions need to spend time guiding students for success in the classroom. Students need encouragement to stay engaged and involved in their learning experiences, campus life, and activities. Students who participate in service activities often earn better grades and are better able to apply skills learned in their courses. Students also form deeper relationships with fellow students and feel more welcomed at the university level. From a faculty and research perspective, researchers worked collaboratively to building trust and engaged in knowledge exchange through various stages throughout the study. The exchange of knowledge helped to shape the research team and enhance collaboration. The importance of building community and fostering relationships can be identified as a step towards reconciliation. ${ }^{[2,9]}$ Sharing knowledge and power is shared equitably between the researchers and the involvement of community members. ${ }^{[9]}$ As in the research study, researchers worked collaboratively to recognize improvements in education from the reflective experiences of the students. In return, these improvements could aim at success in academia and when given opportunities during personal and professional events.

\section{Conflicts of Interest Disclosure}

The author declares that there is no conflict of interest.

\section{REFERENCES}

[1] Howe EC. Saskatchewan with an Aboriginal majority: Education and entrepreneurship. Available from: https://ourspace.uregi na.ca/bitstream/handle/10294/6698/SIPP\%20Public\%20 Policy\%20Papers\%2044.pdf? sequence=1\&isAllowed=y

[2] Gordon CE, White JP. Indigenous educational attainment in Canada. The International Indigenous Policy Journal. 2014; 5(3): 1-28. https://doi.org/10.18584/iipj.2014.5.3.6
[3] Mooring QE. Recruitment, advising, and retention programs - challenges and solutions to the International problem of poor nursing student retention: A narrative literature review. Nurse Education Today. 2016; 40: 204-208. PMid:27125174 https ://doi.org/10.1 $016 / j$.nedt. 2016.03 .003

[4] Ross A. Building on Tinto's model of engagement and persistence: Experiences from the Umthombo Youth Development Foundation Scholarship Scheme. African Journal of Health Professions Edu- 
cation. 2014; 6(2): 119-123. https://doi .org/10.7196/A JHPE. 404

[5] Government of Canada. Truth and reconciliation commission of Canada. 2019. Retrieved from: https://www.rcaanc-cirnac. gc.ca/eng/1450124405592/1529106060525

[6] Botha LR. Using expansive learning to include Indigenous knowledge. Journal of Inclusive Education. 2012; 16(1): 57-70. https: //doi.org/10.1080/13603110903560093

[7] Chilisia B, Tshesko GN. Mixed methods in Indigenous research: Building relationships for sustainable intervention outcomes. Journal of Mixed Methods Research. 2014; 8(3): 222-223. https: //doi.org/10.1177/1558689814527878

[8] Prasad JJ, Showler MB, Ryan AM, et al. When belief precedes being: How attributes and motivation before matriculation lead to fit and academic performance. Journal of Vocational Behavior. 2017; 100: 27-42. https://doi.org/10.1016/j.jvb.2017.02.003

[9] Drawson AS, Toombs E, Mushquash CJ. Indigenous research methods: A systematic review. The International Policy Journal. 2017; 8(2): 1-25. https://doi.org/10.18584/iipj.2017.8.2.5

[10] Le TN, Gobert JM. Translating and implementing a mindfulnessbased youth suicide prevention intervention in a Native American community. Journal of Child and Family Studies. 2015; 24(1): 12-23. https ://doi.org/10.1007/s10826-013-9809-z
[11] Tinto. Leaving College: Rethinking the Causes and Cures of Student Attrition. 2nd ed. Chicago: University of Chicago Press; 1993.

[12] Zimmerman MA. Resiliency Theory: A strengths-based approach to research and practice for adolescent health. Health Education \& Behavior. 2013; 40(4): 381-383. PMid:23863911 https ://doi.or g/10.1177/1090198113493782

[13] Hurd NM, Sanchez B, Zimmerman MA, et al. Natural mentors, racial identity, and educational attainment among African American adolescents: Exploring pathways to success. Child Development. 2012; 83(4): 1196-1212. PMid:22537308 https://doi.org/10.1111/ j.1467-8624.2012.01769.x

[14] Cote-Meek S. Colonized classrooms: Racism, trauma, and resistance in post-secondary education. 1st ed. Winnipeg: Fernwood Publishing; 2014.

[15] Foxall D. Barriers in education of Indigenous nursing students: A literature review. Nursing Praxis in New Zealand. 2013; 29(3): 33-39.

[16] Kukkonen P, Suhonen R, Salminen L. Discontinued students in nursing education-who and why? Nurse Education in Practice. 2016; 17: 67-71. PMid:26774864 https ://doi.org/10.1016/j.nepr .2015 .12 .007

[17] Nygard RH, Saus M. Emphasizing Indigenous communities in social work research ethics. International Social Work. 2016; 59(5): 666-678. https : //doi.org/10.1177/0020872816646815 